\title{
On the Linear Boltzmann Equation with Rough Granular Collisions and Spin
}

\author{
Rolf Pettersson \\ Department of Mathematics, Chalmers University of Technology, SE-412 96 Göteborg, Sweden
}

\begin{abstract}
This paper considers the time- and space-dependent linear Boltzmann equation with general boundary conditions in the case of inelastic rough granular collisions. First, in the angular cut-off case or hard sphere case, mild $L^{1}$-solutions are constructed as limits of the iterate functions and boundedness of higher velocity moments are discussed in the case of hard inverse power collisions or hard sphere collisions. Furthermore, convergence of solutions to a stationary state, when time goes to infinity, is discussed, using a generalized H-theorem.
\end{abstract}

Keywords: linear Boltzmann equation, granular collisions, hard spheres, spine.

PACS: $02.30 . \mathrm{Rz}, 05.20 . \mathrm{Dd}, 45.70 .-\mathrm{n}, 47.45 . \mathrm{Ab}, 51.10 .+\mathrm{y}$.

\section{INTRODUCTION}

The linear Boltzmann equation is frequently used for mathematical modelling in physics, (e.g. for describing the neutron distribution in reactor physics, cf. [1]-[3]). In our earlier papers [4]-[6] we have studied the linear Boltzmann equation (different from the linearized equation), both in the angular cut-off case and the infinite range case, for a function $f(\mathbf{x}, \mathbf{v}, t)$ representing the distribution of particles with mass $m$ undergoing elastic binary collision with other particles with mass $m_{*}$ and with a given (known) distribution function $Y\left(\mathbf{x}, \mathbf{v}_{*}\right)$. In recent years there has been a significant interest in the study of kinetic models for granular flows, mostly with the non-linear Boltzmann equation; see ref. [7] for an overview, with many further references, and also [8]-[9]. Our papers [10]-[14] consider the timedependent and the stationary linear Boltzmann equations for inelastic (granular) collisions, all papers in the angular cut-off case, but the paper [15] studies the (granular) infinite range case.

The purpose of this paper is to generalize our earlier results to the case of rough granular collisions with spin, $\mathrm{cf}$. [16]-[18]. (For details, see our earlier papers, e.g. [10].)

So we will study collisions between particles with mass $m$ and particles with mass $m_{*}$, such that momentum is conserved, $m \mathbf{v}+m_{*} \mathbf{v}_{*}=m \mathbf{v}^{\prime}+m_{*} \mathbf{v}_{*}^{\prime}$, where $\mathbf{v}, \mathbf{v}_{*}$ are velocities before and $\mathbf{v}^{\prime}, \mathbf{v}_{*}^{\prime}$ are velocities after a collision, and such that also the angular momentum for the two particles are conserved, cf. [16]-[18].

In the elastic case, where also kinetic energy is conserved, one finds that the velocities after a binary collision terminate on two concentric spheres, cf Figure 1 in [4].

In the granular, inelastic rough case we assume the following relation between the relative velocity components normal to a plane of contact of the two particles,

$$
\mathbf{w}^{\prime} \cdot \mathbf{u}=-a(\mathbf{w} \cdot \mathbf{u})
$$

where $a$ is a constant, called the normal restitution coefficient, $0<a \leq 1$, and $\mathbf{w}=\mathbf{v}-\mathbf{v}_{*}, \mathbf{w}^{\prime}=\mathbf{v}^{\prime}-\mathbf{v}_{*}^{\prime}$ are the relative velocities before and after the collision, and $\mathbf{u}$ is a unit vector, with $a=1$ in the elastic case.

Furthermore, in the rough granular case, the vector product satisfies the relation

$$
\mathbf{w}^{\prime} \times \mathbf{u}=-b(\mathbf{w} \times \mathbf{u})
$$

with the tangential restitution coefficient $b,-1 \leq b \leq 1$, where $b=-1$ for smooth collisions. Let also the unit vector $\mathbf{u}$ be given in spherical coordinates,

$$
\mathbf{u}=(\sin \theta \cos \phi, \sin \theta \sin \phi, \cos \theta), 0 \leq \theta \leq \pi / 2,0 \leq \phi<2 \pi .
$$

In the case of hard sphere collisions, cf. [18], with for simplicity equal masses $m_{*}=m$, then the pre-collisional contact velocities are

$$
\tilde{\mathbf{v}}=\mathbf{v}-\mathbf{u} \times \mathbf{s}, \quad \tilde{\mathbf{v}}_{*}=\mathbf{v}_{*}+\mathbf{u} \times \mathbf{s}_{*}
$$


and the corresponding relative velocity

$$
\tilde{\mathbf{w}}=\mathbf{w}-\mathbf{u} \times\left(\mathbf{s}+\mathbf{s}_{*}\right)
$$

with spin vectors $\mathbf{s}=\rho \boldsymbol{\omega}, \mathbf{s}_{*}=\rho \boldsymbol{\omega}_{*}$, where $\boldsymbol{\omega}, \boldsymbol{\omega}_{*}$ are the angular velocity vectors and $\rho$ is the radius of the spheres. Then the post-collisional velocities will be, cf. [16]-[18].

$$
\mathbf{v}^{\prime}=\mathbf{v}-\boldsymbol{\delta} \quad \mathbf{v}_{*}^{\prime}=\mathbf{v}_{*}+\boldsymbol{\delta},
$$

where $m \boldsymbol{\delta}$ is the impulse of particle 1 on particle 2 , so the post-collisional relative velocity and contact relative velocities are, cf. [18], $\mathbf{w}^{\prime}=\mathbf{w}-2 \boldsymbol{\delta}$ and

$$
\tilde{\mathbf{w}}^{\prime}=\tilde{\mathbf{w}}-2 \boldsymbol{\delta}+\frac{2}{\kappa}(\mathbf{u} \times(\mathbf{u} \times \boldsymbol{\delta}))
$$

with a constant $\kappa=2 / 5$ for spheres with uniform masses. One finds, cf. [18], that

$$
\boldsymbol{\delta}=\tilde{a}(\mathbf{w} \cdot \mathbf{u}) \mathbf{u}+\tilde{b}\left(\mathbf{w}-(\mathbf{w} \cdot \mathbf{u}) \mathbf{u}-\mathbf{u} \times\left(\mathbf{s}+\mathbf{s}_{*}\right)\right)
$$

with constants $\tilde{a}=(1+a) / 2, \tilde{b}=\frac{\kappa(1+b)}{2(\kappa+1)}$, and finally for the normal and tangential relative velocities after and before collision, that

$$
\mathbf{u} \cdot \tilde{\mathbf{w}}^{\prime}=-\tilde{a}(\mathbf{u} \cdot \tilde{\mathbf{w}}), \quad \mathbf{u} \times \tilde{\mathbf{w}}^{\prime}=-\tilde{b}(\mathbf{u} \times \tilde{\mathbf{w}}) .
$$

Moreover, if we change notations and let ${ }^{\prime} \mathbf{v},{ }^{\prime} \mathbf{v}_{*}$ be the velocities before, and $\mathbf{v}, \mathbf{v}_{*}$ the velocities after a binary rough inelastic collision, then by (8)

$$
{ }^{\prime} \mathbf{v}=\mathbf{v}-\overline{\boldsymbol{\delta}} \quad{ }^{\prime} \mathbf{v}_{*}=\mathbf{v}_{*}+\overline{\boldsymbol{\delta}},
$$

with

$$
\overline{\boldsymbol{\delta}}=\frac{(1+a)}{2 a}(\mathbf{w} \cdot \mathbf{u}) \mathbf{u}+\frac{\kappa(1+b)}{(\kappa+1) 2 b}\left(\mathbf{w}-(\mathbf{w} \cdot \mathbf{u}) \mathbf{u}-\mathbf{u} \times\left(\mathbf{s}+\mathbf{s}_{*}\right)\right)
$$

cf. [18].

\section{PRELIMINARIES}

We consider the time-dependent transport equation for a distribution function $f(\mathbf{x}, \mathbf{v}, \mathbf{s}, t)$, depending on a space variable $\mathbf{x}=\left(x_{1}, x_{2}, x_{3}\right)$ in a bounded convex body $D$ with (piece-wise) $C^{1}$-boundary $\Gamma=\partial D$, and depending on a velocity variable $\mathbf{v}=\left(v_{1}, v_{2}, v_{3}\right) \in V=\mathbb{R}^{3}$, a spin variable $\mathbf{s}=\left(s_{1}, s_{2}, s_{3}\right) \in S$, and a time variable $t \in \mathbb{R}_{+}$. Then the linear Boltzmann equation is in the strong form

$$
\begin{array}{r}
\frac{\partial f}{\partial t}(\mathbf{x}, \mathbf{v}, \mathbf{s}, t)+\mathbf{v} \cdot \operatorname{grad}_{\mathbf{x}} f(\mathbf{x}, \mathbf{v}, \mathbf{s}, t)=(Q f)(\mathbf{x}, \mathbf{v}, \mathbf{s}, t), \\
\mathbf{x} \in D, \quad \mathbf{v} \in V=\mathbb{R}^{3}, \mathbf{s} \in S, \quad t \in \mathbb{R}_{+},
\end{array}
$$

supplemented by initial data

$$
f(\mathbf{x}, \mathbf{v}, \mathbf{s}, 0)=f_{0}(\mathbf{x}, \mathbf{v}, \mathbf{s}), \mathbf{x} \in D, \mathbf{v} \in V, \mathbf{s} \in S .
$$

The collision term can, in the case of inelastic (granular) collision, be written, cf. [7]-[12],

$$
(Q f)(\mathbf{x}, \mathbf{v}, \mathbf{s}, t)=\int_{V} \int_{S} \int_{\Omega}\left[J_{a, b}(\theta, w) Y\left(\mathbf{x}, \mathbf{v}_{*}, \mathbf{s}_{*}\right) f\left(\mathbf{x},{ }^{\prime} \mathbf{v}, ' \mathbf{s}, t\right)-Y\left(\mathbf{x}, \mathbf{v}_{*}, \mathbf{s}_{*}\right) f(\mathbf{x}, \mathbf{v}, \mathbf{s}, t)\right] B(\theta, w) d \mathbf{v}_{*} d \mathbf{s}_{*} d \theta d \phi
$$

with $w=\left|\mathbf{v}-\mathbf{v}_{*}\right|$, where $Y \geq 0$ is a known distribution, $B \geq 0$ is given by the collision process, and finally $J_{a, b}$ is a factor depending on the granular process (and giving mass conservation, if the gain and the loss integrals converge separately). Furthermore, ' $\mathbf{v},{ }^{\prime} \mathbf{v}_{*}$ in (10) are the velocities before and $\mathbf{v}, \mathbf{v}_{*}$ the velocities after the binary collision, cf. (10)-(11), and $\Omega=\{(\theta, \phi): 0 \leq \theta<\hat{\theta}, 0 \leq \phi<2 \pi\}$ represents the impact plane, where $\hat{\theta}<\frac{\pi}{2}$ in the angular cut-off case, and $\hat{\theta}=\frac{\pi}{2}$ in the infinite range case. The collision function $B(\theta, w)$ is in the physically interesting case with inverse $\mathrm{k}$-th power collision forces given by

$$
B(\theta, w)=b(\theta) w^{\gamma}, \quad \gamma=\frac{k-5}{k-1}, w=\left|\mathbf{v}-\mathbf{v}_{*}\right|,
$$


with hard forces for $k>5$, Maxwellian for $k=5$, and soft forces for $3<k<5$, where $b(\theta)$ has a non-integrable singularity for $\theta=\frac{\pi}{2}$, of order $\alpha=-(k+1) /(k-1)$. But in the case of hard sphere collisions, then (for $\left.\gamma=1\right)$ the collision function is given by

$$
B(\theta, w)=\text { const. } w \sin \theta \cos \theta
$$

So in the angular cut-off case one can choose $\hat{\theta}<\frac{\pi}{2}$, and then the gain and the loss terms can be separated $(Q f)(\mathbf{x}, \mathbf{v}, \mathbf{s}, t)=\left(Q^{+} f\right)(\mathbf{x}, \mathbf{v}, \mathbf{s}, t)-\left(Q^{-} f\right)(\mathbf{x}, \mathbf{v}, \mathbf{s}, t)$, where the gain term can be written (with a kernel $\left.K_{a, b}\right)$

$$
\left(Q^{+} f\right)(\mathbf{x}, \mathbf{v}, \mathbf{s}, t)=\int_{V} \int_{S} K_{a, b}\left(\mathbf{x},{ }^{\prime} \mathbf{v},{ }^{\prime} \mathbf{s} \rightarrow \mathbf{v}, \mathbf{s}\right) f\left(\mathbf{x},{ }^{\prime} \mathbf{v},{ }^{\prime} \mathbf{s}, t\right) d^{\prime} \mathbf{v} d^{\prime} \mathbf{s},
$$

and the loss term is written with the collision frequency $L(\mathbf{x}, \mathbf{v}, \mathbf{s})$ as $\left(Q^{-} f\right)(\mathbf{x}, \mathbf{v}, \mathbf{s}, t)=L(\mathbf{x}, \mathbf{v}, \mathbf{s}) f(\mathbf{x}, \mathbf{v}, \mathbf{s}, t)$. In the case of non-absorbing body we have that $L(\mathbf{x}, \mathbf{v}, \mathbf{s})=\int_{V} \int_{S} K_{a, b}\left(\mathbf{x}, \mathbf{v}, \mathbf{s} \rightarrow \mathbf{v}^{\prime}, \mathbf{s}^{\prime}\right) d \mathbf{v}^{\prime} d \mathbf{s}^{\prime}$. Furthermore, equation (12) is in the space-dependent case supplemented by ( general) boundary conditions

$$
\begin{aligned}
& f_{-}(\mathbf{x}, \mathbf{v}, \mathbf{s}, t)=\iint \frac{|\mathbf{n} \cdot \tilde{\mathbf{v}}|}{|\mathbf{n} \cdot \mathbf{v}|} R(\mathbf{x}, \tilde{\mathbf{v}}, \tilde{\mathbf{s}} \rightarrow \mathbf{v}, \mathbf{s}) f_{+}(\mathbf{x}, \tilde{\mathbf{v}}, \tilde{\mathbf{s}}, t) d \tilde{\mathbf{v}} d \tilde{\mathbf{s}}, \\
& \mathbf{n} \cdot \mathbf{v}<0, \mathbf{n} \cdot \tilde{\mathbf{v}}>0, \mathbf{x} \in \Gamma=\partial D, t \in \mathbb{R}_{+}, \mathbf{s} \in S, \tilde{\mathbf{s}} \in S,
\end{aligned}
$$

where $\mathbf{n}=\mathbf{n}(\mathbf{x})$ is the unit outward normal at $\mathbf{x} \in \Gamma=\partial D$. The function $R \geq 0$ satisfies (in the non-absorbing boundary case) $\int_{V} \int_{S} R(\mathbf{x}, \tilde{\mathbf{v}}, \tilde{\mathbf{s}} \rightarrow \mathbf{v}, \mathbf{s}) d \mathbf{v} d \mathbf{s} \equiv 1$, and $f_{-}$and $f_{+}$represent the ingoing and outgoing trace functions corresponding to $f$. In the specular reflection case the function $R$ is represented by a Dirac measure $R(\mathbf{x}, \tilde{\mathbf{v}} \rightarrow \mathbf{v})=$ $\delta(\mathbf{v}-\tilde{\mathbf{v}}+2(\mathbf{n} \cdot \tilde{\mathbf{v}}) \mathbf{n})$, and in the diffuse reflection case $R(\mathbf{x}, \tilde{\mathbf{v}} \rightarrow \mathbf{v})=|\mathbf{n} \cdot \mathbf{v}| W(\mathbf{x}, \mathbf{v})$ with some given function $W \geq 0$, (e.g. Maxwellian function).

Let $t_{b} \equiv t_{b}(\mathbf{x}, \mathbf{v})=\inf _{\tau \in \mathbb{R}_{+}}\{\tau: \mathbf{x}-\tau \mathbf{v} \notin D\}$, and $\mathbf{x}_{b} \equiv \mathbf{x}_{b}(\mathbf{x}, \mathbf{v})=\mathbf{x}-t_{b} \mathbf{v}$, where $t_{b}$ represents the time for a particle going with velocity $\mathbf{v}$ from the boundary point $\mathbf{x}_{b}$ to the point $\mathbf{x}$.

Then, using differentiation along the characteristics, equation (12) can formally be transformed to a mild equation, and also to an exponential form of equation in the angular cut-off or hard sphere cases.

\section{CONSTRUCTION OF SOLUTIONS}

We construct $L^{1}$-solutions to our problems as limits of iterate functions $f^{n}$, when $n \rightarrow \infty$. Let first $f^{-1}(\mathbf{x}, \mathbf{v}, \mathbf{s}, t) \equiv$ 0 . Then define for given $f^{n-1}$ the next iterate $f^{n}, n \geq 0$, first at the ingoing boundary (using the appropriate boundary condition), and then inside $D$ and at the outgoing boundary (using the exponential form of the equation),

$$
\begin{gathered}
f_{-}^{n}(\mathbf{x}, \mathbf{v}, \mathbf{s}, t)=\int_{V} \int_{S} \frac{|\mathbf{n} \cdot \tilde{\mathbf{v}}|}{|\mathbf{n} \cdot \mathbf{v}|} R(\mathbf{x}, \tilde{\mathbf{v}}, \tilde{\mathbf{s}} \rightarrow \mathbf{v}, \mathbf{s}) f_{+}^{n-1}(\mathbf{x}, \tilde{\mathbf{v}}, \tilde{\mathbf{s}}, t) d \tilde{\mathbf{v}} d \tilde{\mathbf{s}} \\
f^{n}(\mathbf{x}, \mathbf{v}, \mathbf{s}, t)=\bar{f}^{n}(\mathbf{x}, \mathbf{v}, \mathbf{s}, t) \exp \left[-\int_{0}^{t} L(\mathbf{x}-\eta \mathbf{v}, \mathbf{v}, \mathbf{s}) d \eta\right] \\
+\int_{0}^{t} \exp \left[-\int_{0}^{\tau} L(\mathbf{x}-\eta \mathbf{v}, \mathbf{v}, \mathbf{s}) d \eta\right] \int_{V} \int_{S} K_{a, b}\left(\mathbf{x}-\tau \mathbf{v},{ }^{\prime} \mathbf{v}, ' \mathbf{s} \rightarrow \mathbf{v}, \mathbf{s}\right) f^{n-1}\left(\mathbf{x}-\tau \mathbf{v},{ }^{\prime} \mathbf{v},{ }^{\prime} \mathbf{s}, t-\tau\right) d^{\prime} \mathbf{v} d^{\prime} \mathbf{s} d \tau,
\end{gathered}
$$

where

$$
\bar{f}^{n}(\mathbf{x}, \mathbf{v}, \mathbf{s}, t)= \begin{cases}f_{0}(\mathbf{x}-t \mathbf{v}, \mathbf{v}, \mathbf{s}), & 0 \leq t \leq t_{b} \\ f_{-}^{n}\left(\mathbf{x}_{b}, \mathbf{v}, \mathbf{s}, t-t_{b}\right), & t>t_{b}\end{cases}
$$

Let also $f^{n}(\mathbf{x}, \mathbf{v}, \mathbf{s}, t) \equiv 0$ for $\mathbf{x} \in \mathbb{R}^{3} \backslash D$. Now we get a strong pointwise monotonicity lemma, $f^{n}(\mathbf{x}, \mathbf{v}, \mathbf{s}, t) \geq$ $f^{n-1}(\mathbf{x}, \mathbf{v}, \mathbf{s}, t)$, which is essential and can be proved by induction.

Then, by differentiation along the characteristics and integration (with Green's formula), we find (using the equations above, cf. [10]), that

$$
\int_{D} \int_{V} \int_{S} f^{n}(\mathbf{x}, \mathbf{v}, \mathbf{s}, t) d \mathbf{x} d \mathbf{v} d \mathbf{s} \leq \int_{D} \int_{V} \int_{S} f_{0}(\mathbf{x}, \mathbf{v}, \mathbf{s}) d \mathbf{x} d \mathbf{v} d \mathbf{s},
$$


so Levi's theorem (on monotone convergence) gives existence of (mild) $L^{1}$-solutions

$$
f(\mathbf{x}, \mathbf{v}, \mathbf{s}, t)=\lim _{n \rightarrow \infty} f^{n}(\mathbf{x}, \mathbf{v}, \mathbf{s}, t)
$$

to our problem with rough granular gases (almost in the same way as for the elastic collision case). Furthermore, if $L(\mathbf{x}, \mathbf{v}, \mathbf{s}) f(\mathbf{x}, \mathbf{v}, \mathbf{s}, t) \in L^{1}(D \times V \times S)$, then we get equality in (22) for the limit function $f$, giving mass conservation together with uniqueness in the relevant function space (cf [4]-[6], [10], [11], and also Proposition 3.3, chapter 11 in $[3])$.

Theorem 1. (Existence)

Assume for inelastic rough granular collisions (or elastic collisions) that the function B is given by (16), or (15) with angular cut-off, and that $K_{a, b}, L$ and $R$ are non-negative, measurable functions, such that $L \in L_{\text {loc }}^{1}(D \times V \times S)$. Then for every $f_{0} \in L^{1}(D \times V \times S)$ there exists a mild $L^{1}$-solution $f(\mathbf{x}, \mathbf{v}, \mathbf{s}, t)$ to the problem (12)-(14) with (18), satisfying the corresponding inequality in (22). Furthermore, if $L f \in L^{1}(D \times V \times S)$, then equality holds in (22) for the limiting function $f$, giving mass conservation together with uniqueness in the relevant function space.

Remark 1 The assumption $L f \in L^{1}(D \times V \times S)$ is, for instance, satisfied for the solution $f$ in the case of hard inverse power forces or hard sphere collisions, together with e.g. specular or diffuse boundary reflections. This follows from a statement on global boundedness (in time) of higher velocity moments, (cf. [12] and [14]), and see also the results in the next section.

Remark 2 There holds also in the granular inelastic collision case an H-theorem for a general relative entropy (Lyapunov) functional, cf [6],

$$
H_{F}^{\Phi}(f)(t)=\int_{D} \int_{V} \int_{S} \Phi\left(\frac{f(\mathbf{x}, \mathbf{v}, \mathbf{s}, t)}{F(\mathbf{x}, \mathbf{v}, \mathbf{s})}\right) F(\mathbf{x}, \mathbf{v}, \mathbf{s}) d \mathbf{x} d \mathbf{v} d \mathbf{s}
$$

giving that this $\mathrm{H}$-functional is non-increasing in time, if $\Phi=\Phi(z), \mathbb{R}_{+} \rightarrow \mathbb{R}$, is a convex $C^{1}$-function, and if there exists a corresponding stationary solution $F(\mathbf{x}, \mathbf{v}, \mathbf{s})$ with the same total mass as the initial data $f_{0}(\mathbf{x}, \mathbf{v}, \mathbf{s})$; cf. Theorem 5.1 in [10]. By using this H-functional one can prove that every time-dependent solution $f(\mathbf{x}, \mathbf{v}, \mathbf{s}, t)$ converges to the corresponding stationary solution $F(\mathbf{x}, \mathbf{v}, \mathbf{s})$, as time goes to infinity; cf. Remark 5.1 in [10] and further references.

\section{BOUNDEDNESS OF HIGHER VELOCITY MOMENTS}

In this section we will generalize a result on global boundedness of higher velocity moments to the case of rough granular collisions with hard potentials or hard spheres. Then we start with some (old) velocity estimates for a binary collision, and also give the corresponding moment estimates, cf. Propositions 1.1 and 1.2 in [4].

Proposition 2. If $\mathbf{v}$ and $\mathbf{v}_{a}^{\prime}(\theta, \phi)$ are the velocities before and after a (granular) binary collision, then, with $w=$ $\left|\mathbf{v}-\mathbf{v}_{*}\right|$,

$$
\left|\mathbf{v}_{a}^{\prime}(\theta, \phi)\right|^{2}-|\mathbf{v}|^{2} \leq 2(a+1) \frac{m_{*}}{m+m_{*}} w \cos \theta\left[3\left|\mathbf{v}_{*}\right|-\frac{m}{m+m_{*}}|\mathbf{v}| \cos \theta\right] .
$$

Proposition 3. If $\sigma>0$, there exist constants $c_{1}>0, c_{2}>0$ (depending on $\sigma, m, m_{*}$ and a) such that

$$
\left(1+\left|\mathbf{v}_{a}^{\prime}(\theta, \phi)\right|^{2}\right)^{\sigma / 2}-\left(1+|\mathbf{v}|^{2}\right)^{\sigma / 2} \leq c_{1} w \cos \theta\left(1+\left|\mathbf{v}_{*}\right|\right)^{\max (1, \sigma-1)}\left(1+|\mathbf{v}|^{2}\right)^{\frac{\sigma-2}{2}}-c_{2} w \cos ^{2} \theta\left(1+|\mathbf{v}|^{2}\right)^{\frac{\sigma-1}{2}}
$$

By using these propositions we have earlier got results on boundedness of higher velocity moments for hard inverse collision forces, $0 \leq \gamma<1$, and also, by using a Jensen inequality to get the analogous results for hard sphere collisions, $\gamma=1$, in the space-dependent case with e.g. specular or diffuse reflection boundary.

We start with an elementary lemma (used in the theorem below) for the velocities in a binary collision, where $v=|\mathbf{v}|, v_{*}=\left|\mathbf{v}_{*}\right|$, and $w=|\mathbf{w}|$, cf. [10].

Lemma 4. For $\gamma \geq 0$ it holds that $-w^{\gamma+1} \leq\left(1+v_{*}\right)^{\gamma+1}-2^{-\gamma}\left(1+v^{2}\right)^{\frac{\gamma+1}{2}}$, where $\mathbf{w}=\mathbf{v}-\mathbf{v}_{*}$ is the relative velocity. 
In the case of rough granular collisions with hard spheres, then the collision change of total (translational and rotational) kinetic energy, i.e. moment of order 2, is given by, cf. [18], $\Delta E=E^{\prime}-E=\Delta E_{t r}+\Delta E_{\text {rot }}$, where

$$
\Delta E_{t r}=-\frac{m}{4}\left(1-a^{2}\right)(\mathbf{u} \cdot \mathbf{w})^{2}
$$

and

$$
\Delta E_{r o t}=-\frac{m}{4} \frac{\kappa\left(1-b^{2}\right)}{1+\kappa}\left(\mathbf{w}-\mathbf{u} \times\left(\mathbf{s}+\mathbf{s}_{*}\right)-(\mathbf{w} \cdot \mathbf{u}) \mathbf{u}\right)^{2}
$$

with the total energy

$$
E=\frac{m}{2} v^{2}+\frac{m}{2} v_{*}^{2}+\frac{I}{2} \omega^{2}+\frac{I}{2} \omega_{*}^{2},
$$

where $\mathbf{s}=\rho \boldsymbol{\omega}$ is the spin variable (and $I$ is the moment inertia).

Then the total energy change in a collision is less or equal to the change of translational energy, $\Delta E \leq \Delta E_{t r}$, so we will study boundedness of higher velocity moments, because $\Delta E_{\text {rot }} \leq 0$.

Now we can formulate our main result on global boundedness (in time) for hard potentials or hard sphere collisions, i.e. with $0 \leq \gamma \leq 1$, in the case of rough granular collisions, together with diffuse or specular reflection boundary conditions, in the following theorem. Compare Theorem 4.1 in [4] for the case of hard inverse forces.

Let the velocity moments (for the iterate $f^{n}$ ) be defined by

$$
M_{\sigma}(t) \equiv M_{\sigma}^{n}(t)=\int_{D} \int_{V} \int_{S}\left(1+v^{2}\right)^{\sigma / 2} f^{n}(\mathbf{x}, \mathbf{v}, \mathbf{s}, t) d \mathbf{x} d \mathbf{v} d \mathbf{s},
$$

and let also (for simplicity) the spin variable be bounded.

Theorem 5. Assume for rough granular collisions with hard potentials or hard spheres, $0 \leq \gamma \leq 1$, that the function $B(\theta, w)$ is given by equation (15) or (16), and suppose that the function $Y\left(\mathbf{x}, \mathbf{v}_{*}, \mathbf{s}_{*}\right)$ satisfies the following conditions:

$$
\begin{aligned}
& \int_{V}\left(1+v_{*}\right)^{\gamma+\max (2, \sigma)} \sup _{\mathbf{x} \in D} Y\left(\mathbf{x}, \mathbf{v}_{*}, \mathbf{s}_{*}\right) d \mathbf{v}_{*}<\infty \\
& \int_{V} \inf _{\mathbf{x} \in D} Y\left(\mathbf{x}, \mathbf{v}_{*}, \mathbf{s}_{*}\right) d \mathbf{v}_{*}>0 .
\end{aligned}
$$

Let the boundary conditions (18) be given by specular or diffuse (Maxwell) reflections.

Then the higher velocity moments belonging to the mild solution $f(\mathbf{x}, \mathbf{v}, \mathbf{s}, t)$ given by (23) are all bounded (globally in time),

$$
\int_{D} \int_{V} \int_{S}\left(1+v^{2}\right)^{\sigma / 2} f(\mathbf{x}, \mathbf{v}, \mathbf{s}, t) d \mathbf{x} d \mathbf{v} d \mathbf{s} \leq C_{\sigma}<\infty, \quad \sigma>0, t>0,0<a \leq 1,-1 \leq b \leq 1
$$

if $\left(1+v^{2}\right)^{\sigma / 2} f_{0}(\mathbf{x}, \mathbf{v}, \mathbf{s}) \in L^{1}(D \times V \times S)$, and the spin variable $\mathbf{s} \in S$ is bounded.

Sketch of proof: Start from the definition of the iterate function $f^{n}(\mathbf{x}, \mathbf{v}, \mathbf{s}, t)$ in equations (19)-(21), and differentiate along the characteristics, using the corresponding mild form of the equation, and then multiply by $\left(1+v^{2}\right)^{\sigma / 2}$, where $v=|\mathbf{v}|, \sigma>0$. Continuing in the same manner as in our earlier papers, see [12] and [14], where we used a Jensen inequality in the case of hard spheres (for $\gamma=1$ ), then the theorem follows, if $n \rightarrow \infty$.

\section{CONCLUSIONS}

In the paper we generalize our earlier results for the Boltzmann equation on elastic or smooth granular collision (of two different particles) to the case of rough granular collisions with spin. As a background we first study hard sphere collisions, cf. ref. [16]-[18] for details. In our method construction of iterate functions, eq. (19)-(21), is essential, giving a monotonicity lemma, which can be proved by induction. Then we get an existence result for our linear Boltzmann equation with spin (almost in the same manner as in our earlier theorems) using Levi's theorem on monotone convergence. And we also get massconservation and uniqueness in the case of specular or diffuse (Maxwell) boundary conditions, cf. [12] and [14]. Finally we study global boundedness in time for higher velocity moments in the case of hard potentials or hard sphere collisions, using our old estimates for binary collisions (of elastic or granular smooth particles). We also discuss the use of H-theorem for studying convergence to a stationary solution. To summarizing, our earlier results for smooth granular collisions are generalized to rough collisions in a model with similar collision functions. 


\section{REFERENCES}

1. N. Bellomo, A. Palczewski, and G. Toscani, Mathematical Topics in Nonlinear Kinetic Theory, World Scientific, 1989.

2. C. Cercignani, The Boltzmann Equation and its Applications, Springer-Verlag, 1988.

3. W. Greenberg, C. van der Mee and V. Protopopescu, Boundary Value Problems in Abstract Kinetic Theory, Birkhäuser-Verlag, 1987.

4. R. Pettersson, IMA J. Appl. Math. 38, 151-166 (1987).

5. R. Pettersson, J. Stat. Phys. 59, 403-440 (1990).

6. R. Pettersson, J. Stat. Phys. 72, 355-380 (1993).

7. C. Villani, J. Stat. Phys. 124, 781-822 (2006).

8. J. J. Brey, J. W. Dufty and A. Santos, J. Stat. Phys. 87, 1051-1068 (1997).

9. J. J. Brey, J. W. Dufty and A. Santos, J. Stat. Phys. 97, 281-322 (1999).

10. R. Pettersson, Transp. Th. Stat. Phys. 33, 527-543 (2004).

11. R. Pettersson, AIP Conf. Proc. 762, RGD24, 252-257 (2005).

12. R. Pettersson, Il Nuovo Cimento 33, 189-197 (2010).

13. R. Pettersson, AIP Conf. Proc. 1333, RGD27, 111-116 (2011).

14. R. Pettersson, to appear in Transp. Th. Stat. Phys. (2012).

15. R. Pettersson, AIP Conf. Proc. 1084, RGD26, 135-139 (2008).

16. A. Santos, G. M. Kremer and V. Garzó, Prog. Th. Phys. Suppl. 184, 33-48 (2010).

17. A. Santos, AIP Conf. Proc. 1333, RGD27, 41-48 (2011).

18. A. Santos, G. M. Kremer and M. dos Santos, Phys. Fluids 23, 030604 (2011). 